\title{
Antibacterial Effect of Crude Extracts of Kaempferia parviflora (Krachaidam) against Cronobacter spp. and Enterohemorrhagic Escherichia coli (EHEC) in Various Dairy Foods: A Preliminary Study
}

\author{
'Dana Jeong1, 'Dong-Hyeon Kim", 'Jung-Whan Chon ${ }^{1,2}$, 'Hyunsook Kim³, Soo-Kyung Lee', \\ Hong-Seok Kim${ }^{1}$, Jin-Hyuk Yim ${ }^{1}$, Kwang-Young Song ${ }^{1 *}$, Il-Byung Kang ${ }^{1}$, Young-Ji Kim¹, \\ Jin-Hyeong Park', Ho-Seok Jang ${ }^{1}$, Soo-Hyun Kang ${ }^{4},{ }^{\dagger}$ Soo-Ki Kim ${ }^{4}$ and ${ }^{\dagger}$ Kun-Ho Seo ${ }^{1}$ \\ ${ }^{1}$ Center for One Health, College of Veterinary Medicine, Konkuk University, Seoul 05029, Korea \\ ${ }^{2}$ National Center for Toxicological Research, US Food and Drug Administration, Jefferson, AR 72079, USA \\ ${ }^{3}$ Dept. of Food \& Nutrition, College of Human Ecology, Hanyang University, Seoul 04763, Korea \\ ${ }^{4}$ Dept. of Animal Science \& Technology, College of Animal Bioscience \& Technology,
}

Konkuk University, Seoul 05029, Korea

\begin{abstract}
Rhizomes of Kaempferia parviflora (Zingiberaceae) have been used in traditional Thai medicine for health promotion. In this study, the antibacterial activity of ethanol extract of $K$. parviflora against Cronobacter spp. and enterohemorrhagic Escherichia coli (EHEC) was investigated using paper disc dilution method. The results revealed that the ethanol extract exhibited antibacterial activity against Cronobacter spp. and EHEC. With an increasing concentration of $K$. parviflora ethanol extract, larger zones of inhibition of Cronobacter spp. and EHEC strains tested were observed. Therefore, its antibacterial activity suggested that $K$. parviflora could be used as a natural additive to ascertain food safety of various dairy products.

Keywords: Kaempferia parviflora (Krachaidam), Cronobacter spp., enterohemorrhagic Escherichia coli (EHEC), antibacterial activity
\end{abstract}

\section{Introduction}

Medicinal plants are useful alternative source of novel drugs containing various pharmacological activities such as antimicrobial activities, anti-inflammatory, and so on (Welbat et al., 2016). Kaempferia parviflora Wall. Ex Baker (Kaempferia parviflora) or Kra-chai-dam belonging to the Zingiberaceae family is one of the attractive plants for the reasons of various favorable activity (Rujjanawate et al., 2005) (Fig. 1). It has long been used in Thai traditional medicine for antioxidant, antiallergic, antitumor, cardioprotective, allergic, and so on. Furthermore,

\footnotetext{
${ }^{+}$These authors contributed equally to this study.

* Corresponding author: Kwang-Young Song, Center for One Health, College of Veterinary Medicine, Konkuk University, Seoul 05029, Korea. Tel: +82-2-450-4121, Fax: +82-2-3436-4128, E-mail: drkysong@gmail.com
}

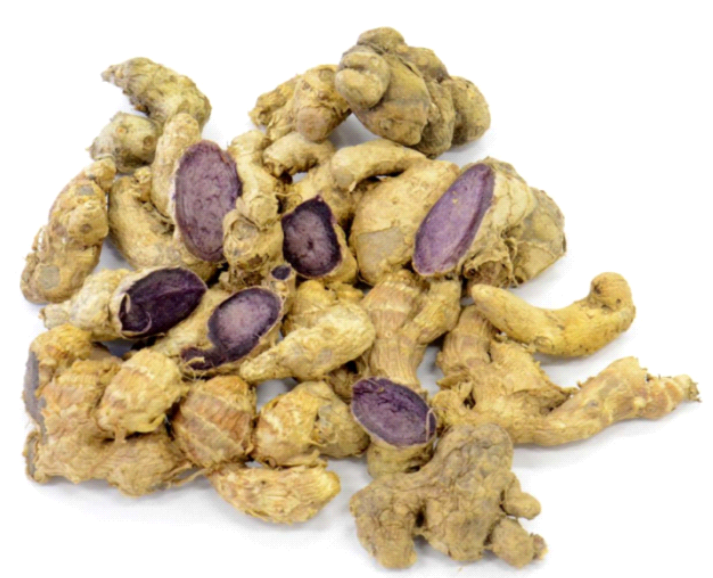

Fig. 1. Kaempferia parviflora (Krachai Dam) is a member of the ginger family and is known in Thailand as Thai ginseng or Black Ginger (Yoshino et al., 2014) 
the extracts and flavone derivatives from the rhizome of Kaempferia parviflora could suppress function of multidrug resistance associated-proteins (MRP), in addition to P-glycoprotein, in cancer cells (Patanasethanont et al., 2006; Patanasethanont et al., 2007).

Also, pharmacological studies have shown that the extract of Kaempferia parviflora rhizomes has antigastric ulcer, antimicrobacterial, and antiviral effects (Rujjanawate et al., 2005: Sookkongwaree et al., 2006). Horigome et al. (2014) reported that Kaempferia parviflora could modulate the expression of multidrug resistance associated-proteins, because Kaempferia parviflora contains 11 polymethoxyflavonoids, 7 of which have been identified as possessing inhibitory effects (Table 1).

Recently, the bacteria, Cronobacter sakazakii is ubiquitous in nature, but can cause severe illness or death in newborn babies if it is ingested. The most likely cause for an infection is through contaminated powdered infant formula (PIF) or if the bacteria are present in the water used to mix PIF (Bowen and Braden, 2006). Because Cronobacter sakazakii is found frequently in nature, it is important to sterilize both bottles and the water used to make the formula (FAO/WHO, 2006). Before 2007, this Gram negative rod was known as Enterobacter sakazakii. While the organism has been found in other foods, only PIF has been linked to disease outbreaks, since PIF is not a sterile

Table 1. Various components extracted from Kaempferia parviflora as Thai Ginseng

\begin{tabular}{|c|c|c|}
\hline \multicolumn{2}{|r|}{ Components } & Amounts $(\%)$ \\
\hline \multirow{3}{*}{ The three 'main' methoxyflavonoids } & $\triangleright$ 5,7-dimethoxyflavone (DMF) & 0.289 \\
\hline & $\triangleright$ 5,7,4'-trimethoxyflavone (TMF) & 0.0101 \\
\hline & $\triangleright$ 3,5,7,3',4'-pentamethoxyflavone (PMF) & - \\
\hline \multicolumn{2}{|c|}{$\triangleright$ A variety of other polymethoxyflavonoids } & - \\
\hline \multicolumn{2}{|c|}{$\begin{array}{l}\text { 5-hydroxy-3,7-dimethoxyflavone (a methoxyflavone that appears to be well reseached but is not one of the 'primary' } \\
\text { three) }\end{array}$} & 0.0252 \\
\hline \multirow{2}{*}{ Derivatives of kaempferol } & $\triangleright 3,7,4^{\prime}$-trimethylkaempferol & 0.0719 \\
\hline & $\triangleright$ Tetramethylkaempferol & 0.0070 \\
\hline \multirow{2}{*}{ Methylated derivatives of apigenin } & $\triangleright 7,4^{\prime}$-dimethylapigenin & 0.0453 \\
\hline & $\triangleright$ Trimethylapigenin & 1.29 \\
\hline Methylated derivative of quercetin & $\triangleright$ Pentamethylquercetin & 0.391 \\
\hline Derivative of luteolin & $\triangleright$ Tetramethylluteolin & 0.0312 \\
\hline Methoxy derivative of chrysin & $D$ Techtochrysin & 0.131 (dry weight) \\
\hline \multicolumn{2}{|c|}{$\triangleright$ A thermostable lectin (sugar-binding protein) in the rhizome } & 0.45 \\
\hline \multicolumn{2}{|l|}{$\triangleright$ Ayanin } & 0.0111 \\
\hline \multicolumn{2}{|l|}{$\triangle$ Retusine } & 0.0215 \\
\hline \multirow[t]{2}{*}{ Ethanol acetate fragment } & $\triangleright(2 \mathrm{R}, 3 \mathrm{R})-(-)$-aromadendrin trimethyl ether & - \\
\hline & $\triangleright$ Syringetin 3-O-rutinoside & - \\
\hline \multirow[t]{2}{*}{ Methanolic fragment } & $\triangleright$ Tamarixetin 3-O-rutinoside & - \\
\hline & $\triangleright$ Tilianine & - \\
\hline \multicolumn{2}{|c|}{$\triangleright$ 2,4,6-trihydroxyacetophenone 2,4-di-O- $\beta$-D-glucopyranoside } & - \\
\hline \multicolumn{2}{|c|}{$D$ Adenosine } & - \\
\hline \multicolumn{2}{|c|}{$\begin{array}{l}D \text { Kaempferiaosides A and B with similar glycosides as well as kaempferiaosides C and D (flav-3-en-3-ol glycosides) } \\
\text { as well as E and F (acetophenone glycosides) }\end{array}$} & - \\
\hline \multicolumn{2}{|l|}{$\triangleright$ L-phenylalanine } & - \\
\hline \multicolumn{2}{|c|}{ 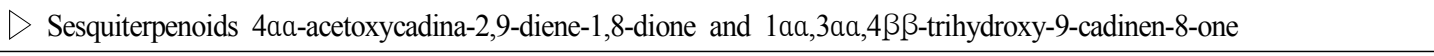 } & - \\
\hline
\end{tabular}

Resources; Yenjai et al., 2004; Sookkongwaree et al., 2006; Patanasethanont et al., 2007; Leardkamolkarn et al., 2009; Horigome et al., 2014; Yoshino et al., 2014. 
product (FAO/WHO, 2008). The organism is unusually resistant to desiccation and can be viable for years in a dried state. Premature, low weight and immune compromised infants are at a higher risk of infection. The US Centers for Disease Control and Prevention (CDC) states that typically four to six cases of infant infections with Cronobacter sakazakii occur each year, however in 2011, there were twelve. It is estimated that 40 to $80 \%$ of the cases will be fatal (FAO/WHO, 2008). And furthermore, Enterohemorrhagic Escherichia coli (EHEC) serotype O157: $\mathrm{H} 7$ is a human pathogen responsible for outbreaks of bloody diarrhea and hemolytic uremic syndrome (HUS) worldwide (Gu et al., 2011). Characteristics of E. coli serotype O157:H7 (EHEC) infection includes abdominal cramps and bloody diarrhea, as well as the life-threatening complication hemolytic uremic syndrome (HUS) (GU et al., 2011; Goldwater and Bettelheim, 2012). Especially, EHEC is often isolated from dairy farms and feedlots, including from house flies, although the latter is not necessarily a mechanical vector (Rahn et al., 1997). Currently no treatment is available for EHEC infections (Goldwater and Bettelheim, 2012). The use of conventional antibiotics exacerbates Shiga toxin-mediated cytotoxicity. In an epidemiology study conducted by the US CDC, patients treated with antibiotics for EHEC enteritis had a higher risk of developing HUS (Goldwater and Bettelheim, 2012).

Until now, there was no report about the inhibition against Cronobacter spp. and Enterohemorrhagic Escherichia coli (EHEC) using the crude extracts from Kaempferia parviflora. Therefore, this study was aimed at investigating the antibacterial activity of Kaempferia parviflora on Cronobacter spp. and Enterohemorrhagic Escherichia coli (EHEC) so as to keep the safety of various dairy foods such as natural additives.

\section{Materials and Methods}

\section{Plant materials and extraction}

Kaempferia parviflora were donated from Department of Animal Science \& Technology, College of Animal Bioscience \& Technology, Konkuk University in Seoul, Korea. The dried plant rhizome powder was macerated in $95 \%$ ethanol for 48 hours with occasionally stirring at room temperature. And then, the soluble ingredients were concentrated by rotary evaporator (RE801C-W, Yamato, JAPAN) at $50^{\circ} \mathrm{C}$ until dryness. The yield were obtained for ethanol extraction type. These stock solutions were filtrated through $0.2 \mathrm{~mm}$ Millipore and stored at $-20^{\circ} \mathrm{C}$ before use.

\section{Bacterial strains and culture condition}

Strains of Cronobacter spp. (C. sakazakii) and Enterohemorrhagic Escherichia coli (EHEC) were obtained from Department of Public Health, College of Veterinary, Konkuk University in Seoul, Korea. Cells were grown on nutrient agar (NA) (Oxoid, UK) overnight. Colonies were transferred into tubes containing cryopreservation fluid according to the instruction of the manufacturer (Original Microbiology Bead Storage System, STS, Technical Service Consultants Limited, UK). The beads were stored at $-70^{\circ} \mathrm{C}$ until use.

\section{Antibacterial susceptibility testing}

The antibacterial activity of $K$ Kaempferia parviflora were tested on Cronobacter spp. and Enterohemorrhagic Escherichia coli (EHEC) using paper disk diffusion inhibition test. In paper disc diffusion test, sterile paper was soaked in the extract of Kaempferia parviflora for $2 \mathrm{~h}$. One fifth milliliter of a $24 \mathrm{~h}$ broth culture $\left(10^{6} \mathrm{cfu} / \mathrm{mL}\right)$ of the bacteria was spread on the surface of gelled sterile Mueller-Hinton agar plates. The paper discs containing the extracts of Kaempferia parviflora (negative control, low, medium, and high concentration) were placed at different areas on the surface of each plate. The extract against the test bacteria was indicated by growth-free "zone of inhibition" near the respective disc.

\section{Statistical analysis}

All experiments were carried out independently in triplicate experiments. The inhibition of various concentration of Kaempferia parviflora ethanol extracts against Cronobacter spp. (C. sakazakii) and Enterohemorrhagic Escherichia coli (EHEC) were evaluated by one way analysis of variance (ANOVA). Statistical significance was accepted at the $P=0.05$ level.

\section{Results and Discussion}

The ethanol extract of Kaempferia parviflora showed various levels of antibacterial activity when tested by paper disc diffusion method (Fig. 2 and 3). This results obtained showed that ethanol extract exhibited antibacterial activities against Cronobacter spp. and Enterohemorrhagic Escherichia coli (EHEC). With increasing concentration of Kaempferia parviflora's ethanol extracts, the results produced the larger zones of inhibition against the 
bacteria tested $(P<0.05)$ (Fig. 2 and 3).

In fact, by this time, several natural materials have been studied for developing natural additives having antibacterial activity by many researchers so as to replace artificial chemical

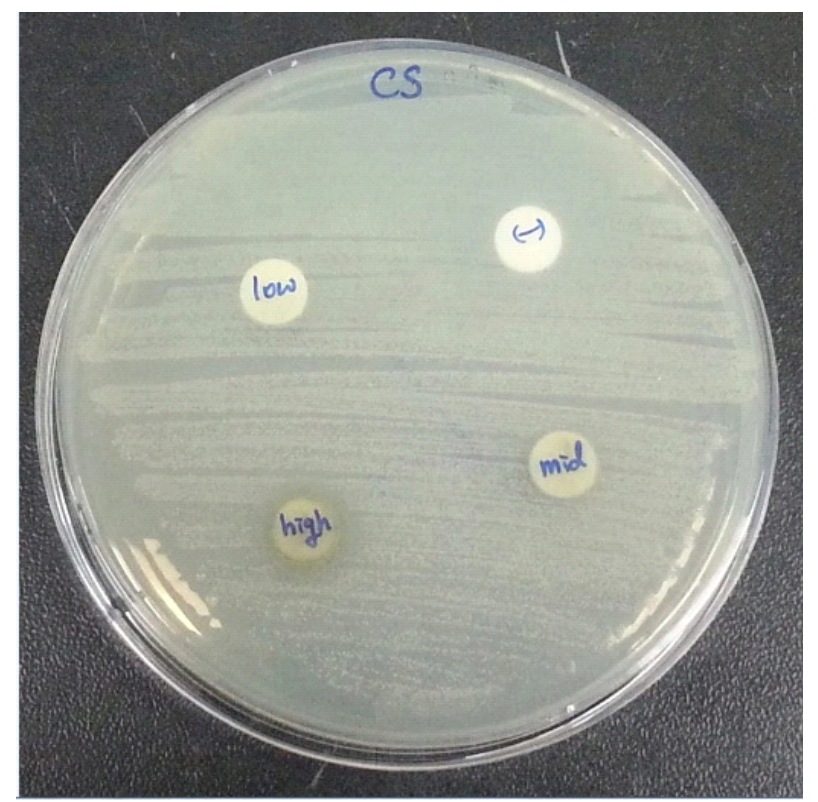

Fig. 2. The antibacterial activity on various concentration of Kaempferia parviflora's ethanol extracts against Cronobacter spp. tested by paper disc diffusion method.

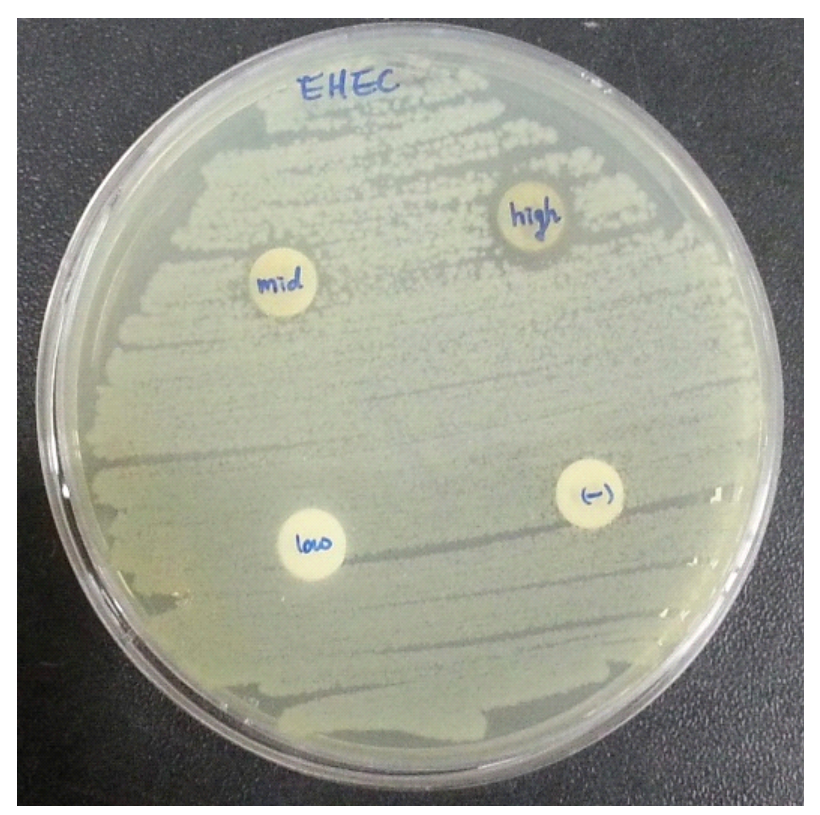

Fig. 3. The antibacterial activity on various concentration of Kaempferia parviflora's ethanol extracts against EHEC tested by paper disc diffusion method. agents. Especially, the efficacy comparison of natural materials was carried out by solvent extraction (methanol, ethanol, water and so on). For example, according to the report of Chaichanawongsaroj et al. (2010), four parts of Kaempferia parviflora's extracts (1) volatile oil, (2) hexane, (3) ethyl acetate and (4) methanol - were screened in vitro for the anti-Helicobacter pylori actions. The strongest growth inhibition against all thirteen $H$. pylori tested was ethyl acetate extract with the minimum inhibitory concentration (MIC) of $32 \mathrm{mg} / \mathrm{mL}$, and the MIC of the extracts from hexane and methanol were $64 \mathrm{mg} / \mathrm{mL}$, while, the MICs of volatile oil was $1,024 \mathrm{mg} / \mathrm{mL}$ (Chaichanawongsaroj et al., 2010). And Mbata et al. (2008) reported that the crude extract from Camellia sinensis by methanol and water exhibited antibacterial activities against L. monocytogenes. The methanol extracts of Camellia sinensis showed larger zones of inhibition against $L$. monocytogenes than the water extract, and also MIC for the water and methanol leaf extract was 0.69 and 0.26 $\mathrm{mg} / \mathrm{mL}$, respectively (Mbata et al., 2008).

And furthermore, Kaempferia parviflora had been reported to possess (1) anti-allergic activities, (2) anti-peptic ulcer, (3) anti-viral proteases, (4) anti-inflammatory activity, (5) anti-cancer action against human cholangiocarcinoma, (6) the increasing mating behavior of the rats via the increasing blood flow to the testicular vessel, (7) the suppressing HFD(high fat diet)-induced obesity through increased energy metabolism, (8) the potential for a supplemental use in vascular endothelial health promotion, and so on (Rujjanawate et al., 2005; Sookkongwaree et al., 2006; Tewtrakul and Subhadhirasakul, 2007; Wattanapitayakul et al., 2007; Tewtrakul et al., 2008; Leardkamolkarn et al., 2009; Sae-wong et al., 2009; Trisomboon, 2009; Yoshino et al., 2014). In general, it was suggested that some bioactive flavonoids containing in the rhizome of Kaempferia parviflora was the major active compound comprising of 5-hydroxy-3,7-dimethoxyflavone, 5-hydroxy-7-methoxyflavone, 5-3,7,4'-trimethoxyflavone, 5-hydroxy-7,4'-dimethoxyflavone, 5-hydroxy-3,7,3'4'-etramethoxyflavone, 3,5,7-trimethoxyflavone, 3,5,7,4'-tetramethoxyflavone, 5,7,4'-trimethoxyflavone and 5,7,3'4'-tetramethoxyflavone (Yenjai et al., 2004; Trisomboon, 2009) (Table 1). For example, Bae et al. (1999) reported that some flavonoids derivatives and their metabolite such as cabreuvin, genistein, hesperitin, irisolidone, and ponciretin displayed anti-Helicobacter pylori activity.

In fact, to date, it has been found that the more functions of Kaempferia parviflora. Wattanathorn et al. (2007) reported Kaempferia parviflora had the activities of anxiolytic and 
antidepression. And recently, Horigome et al. (2014) identified 5,7-dimethoxyflavone (DMF), 5-hydroxy-3,7,3',4'- tetramethoxyflavone (TMF), estimated 3,5,7-trimethoxyflavone, 5-hydroxy-7,4'-dimethoxyflavone, 3,5,7,4'-tetramethoxyflavone by using GC-MS and LC-MS, and investigated their anti-inflammatory effects in rat basophilic leukemia (RBL-2H3) cells stimulated with an $\operatorname{IgE}$ antigen or a calcium ionophore. These results suggested that unknown components within only the $50 \%$ ethanol extract from Kaempferia parviflora may possess the anti-inflammatory effects (Horigome et al., 2014). For getting more effective materials extracted from Kaempferia parviflora, it should be carried out comparative experiments of selecting the best from a variety of extraction solutions.

Hence, from now on, it will be conducted studies actively on many natural materials which have various bioactive functions including antibacterial activity as well as prompting human's health.

In conclusion, the present study demonstrated the potential of Kaempferia parviflora to inhibit the growth of Cronobacter spp. and Enterohemorrhagic Escherichia coli (EHEC) as antimicrobial activity. Therefore, Kaempferia parviflora could be served as the natural resource to develop the antimicrobial agents. However, it needs further investigation to fully understand the precise underlying mechanism including various functions of the crude extract from Kaempferia parviflora (Krachaidam) is warranted, and furthermore, to obtain the safety so as to use natural additional agents for various dairy foods.

\section{Acknowledgement}

This work was supported by the National Research Foundation of Korea (NRF) grant funded by the Korea government (MSIP) (No. 2015R1A2A2A05001288).

Disclaimer: The views expressed herein do not necessarily reflect those of the US Food and Drug Administration or the US Department of Health and Human Services.

\section{References}

1. Bae, E. A., Han, M. J. and Kim, D. H. 1999. In vitro antiHelicobacter pylori activities of some flavonoids and their metabolites. Planta. Med. 65:442-443.

2. Bowen, A. B. and Braden, C. R. 2006. Invasive Enterobacter sakazakii disease in infants. Emerg. Infect. Dis. August Publication http:/www.cdc.gov/ncidod/EID/vol12no08/051509.htm

3. Chaichanawongsaroj, N., Amonyingcharoen, S., Saifah, E., and Poovorawan, Y. 2010. The effects of Kaempferia parviflora on antiinternalization activity of Helicobacter pylori to HEp-2 cells. African Journal of Biotechnology 9:4796-4801.

4. Food and Agricultural Organization of the United Nations (FAO)/World Health Organization (WHO). 2006. Enterobacter sakazakii and Salmonella in powdered infant formula: Meeting report. Microbiological Risk Assessment Series 10 http://www.fao.org/ag/agn/agns/jemra_riskassessment_enter obacter_en.asp

5. Food and Agricultural Organization of the United Nations (FAO)/World Health Organization (WHO). 2008. Enterobacter sakazakii (Cronobacter spp.) in powdered follow-up formulae: Meeting report. Microbiological Risk Assessment Series 15 http://www.fao.org/ag/agn/agns/jemra_riskassessment_enter obacter_en.asp

6. Goldwater, P. N. and Bettelheim, K. A. 2012. Treatment of enterohemorrhagic Escherichia coli (EHEC) infection and hemolytic uremic syndrome (HUS). BMC Medicine 10:12.

7. Gu, J., Ning, Y., Wang, H., Xiao, D., Tang, B., Luo, P., Cheng, Y., Jiang, M., Li, N., Zou, Q. and Mao, X. 2011. Vaccination of attenuated EIS-producing Salmonella induces protective immunity against enterohemorrhagic Escherichia coli in mice. Vaccine 29:7395-7403.

8. Horigome, S., Yoshida, I., Tsuda, A., Harada, T., Yamaguchi, A., Yamazaki, K., Inohana, S., Isagawa, S., Kibune, N., Satoyama, T., Katsuda, S., Suzuki, S., Watai, M., Hirose, N., Mitsue, T., Shirakawa, H. and Komai, M. 2014. Identification and evaluation of anti-inflammatory compounds from Kaempferia parviflora. Biosci. Biotechnol. Biochem. 78:851-860.

9. Leardkamolkarn, V., Tiamyuyen, S. and Sripanidkulchai, B. O. 2009. Pharmacological activity of Kaempferia parviflora extract against human bile duct cancer cell lines. Asian. Pac. J. Cancer. Prev. 10:695-698.

10. Mbata, T. I., Debiao, L. U. and Saikia, A. 2008. Antibacterial activity of the crude extract of Chinese green tea (Camellia sinensis) on Listeria monocytogenes. African Journal of Biotechnology 7:1571-1573.

11. Patanasethanont, D., Nagai, J., Matsuura, C., Fukui, K., 
Sutthanut, K., Sripanidkulchai, B. O., Yumoto, R. and Takano, M. 2007. Modulation of function of multidrug resistance associated-proteins by Kaempferia parviflora extracts and their components. Eur. J. Pharmacol. 566:67-74.

12. Patanasethanont, D., Nagai, J., Yumoto, R., Murakami, T., Sutthanut, K., Sripanidkulchai, B. O., Yenjai, C. and Takano, M. 2006. Effects of Kaempferia parviflora extracts and their flavone constituents on P-glycoprotein function. J. Pharm. Sci. 96:223-233.

13. Rahn, K., Renwick, S. A., Johnson, R. P., Wilson, J. B., Clarke, R. C., Alves, D., McEwen, S., Lior, H. and Spika, J. 1997. Persistence of Escherichia coli O157:H7 in dairy cattle and the dairy farm environment. Epidemiol. Infect. 119:251-259.

14. Rujjanawate, C., Kanjanapothi, D., Amornlerdpison, D. and Pojanagaroon, S. 2005. Anti-gastric ulcer effect of Kaempferia parviflora. J. Ethnopharmacol. 102:120-122.

15. Sae-wong, C., Tansakul, P. and Tewtrakul, S. 2009. Antiinflammatory mechanism of Kaempferia parviflora in murine macrophage cells (RAW 264.7) and in experimental animals. J. Ethnopharmacol. 124:576-580.

16. Sookkongwaree, K., Geitmann, M., Roengsumran, S., Petsom, A. and Danielson, U. H. 2006. Inhibition of viral proteases by Zingiberaceae extracts and flavones isolated from Kaempferia parviflora. Pharmazie 61:717-721.

17. Tewtrakul, S. and Subhadhirasakul, S. 2007. Anti-allergic activity of some selected plants in the Zingiberaceae family. J. Ethnopharmacol. 109:535-538.

18. Tewtrakul, S., Subhadhirasakul, S. and Kummee, S. 2008. Anti-allergic activity of compounds from Kaempferia parviflora. J. Ethnopharmacol. 116:191-193.
19. Trisomboon, H. 2009. Kaempferia parviflora, a Thai herbal plant, neither promote reproductive function nor increase libido via male hormone. Thai Journal of Physiological Sciences 21:83-86.

20. Wattanathorn, J., Pangpookiew, P., Sripanidkulchai, K., Muchimapura, S. and Sripanidkuchai, B. 2007. Evaluation of the anxiolytic and antidepressant effects of alcoholic extract of Kaempferia parviflora in aged rats. Am. J. Agri. Biol. Sci. 2:94-98.

21. Wattanapitayakul, S. K., Suwatronnakorn, M., Chularojmontri, L., Herunsalee, A., Niumsakul, S., Charuchongkolwongse, S. and Chansuvanich, N. 2007. Kaempferia parviflora ethanolic extract promoted nitric oxide production in human umbilical vein endothelial cells. Journal of Ethnopharmacology 110:559-562.

22. Welbat, J. U., Chaisawang, P., Chaijaroonkhanarak, W., Prachaney, P., Pannangrong, W., Sripanidkulchai, B., Wigmore, P. 2016. Kaempferia parviflora extract ameliorates the cognitive impairments and the reduction in cell proliferation induced by valproic acid treatment in rats. Ann. Anat. 206:7-13.

23. Yenjai, C., Prasanphen, K., Daodee, S., Wongpanich, V. and Kittakoop, P. 2004. Bioactive flavonoids from Kaempferia parviflora. Fitoterapia, 75:89-92.

24. Yoshino, S., Kim, M., Awa, R., Kuwahara, H., Kano, Y. and Kawada. T. 2014. Kaempferia parviflora extract increases energy consumption through activation of BAT in mice. Food Science \& Nutrition 2:634-637.

Received April 30, 2016 Revised May 11, 2016 Accepted May 31, 2016 